\title{
Tackling the unique challenges of capacity planning for autologous cell therapies
}

Ching Lam ${ }^{1}$, Edward Meinert ${ }^{2,4}$, Aidong Yang ${ }^{3}$, Zhanfeng Cui ${ }^{{ }^{*}}$

${ }^{1}$ Institute of Biomedical Engineering, Department of Engineering Science, University of Oxford, OX3

7DQ; UK

${ }^{2}$ Department of Paediatrics, University of Oxford, Oxford, OX3 9DU; UK

${ }^{3}$ Department of Engineering Science, University of Oxford, OX1 3PJ; UK

${ }^{4}$ Department of Primary Care and Public Health, Imperial College London, W11 3SP; UK

*Correspondence: zhanfeng.cui@eng.ox.ac.uk

Institute of Biomedical Engineering, Department of Engineering Science, University of Oxford, OX3 7DQ; UK

Capacity planning has been a long-standing issue in the pharmaceutical industry. Setting up Good Manufacturing Practices (GMP) compliant facilities is time-consuming and capital-intensive ${ }^{1}$, but for products to be able to generate maximal economic returns during the market exclusivity period, timely availability of manufacturing capacity is vital. Capacity crunches faced by the monoclonal antibody (mAb) industry in the 1990s were estimated to have caused around \$3-\$4 billion losses in total revenue due to insufficient product supply ${ }^{2,3}$. In addition, the uncertainties in clinical trial outcomes, market pressures, and regulations ${ }^{4}$ make the multi-objective optimisation of capacity planning and product development decisions critical for the industry.

Previous studies have considered the optimisation of investment strategies for product development ${ }^{5}$, manufacturing facility investment decisions and global multi-site supply chain optimisation ${ }^{6}$ for drugs and multi-site continuous bioprocess optimisation for biologics ${ }^{7}$. As more advanced therapeutic medicinal products (ATMPs) move from bench to clinic, the unique challenges for autologous cell therapies present a new problem in the capacity planning domain. Firstly, the unique requirement for autologous cell therapies to be produced on-demand with cell material originating from patients have proven to be commercially challenging ${ }^{8}$. Secondly, with geographical and supply chain constraints, companies are considering building multiple facilities in different regions or finding alternative arrangements such as licensing and outsourcing ${ }^{9}$. Thirdly, fast-track regulatory frameworks introduced by various regulatory authorities are improving the speed-to-market ${ }^{13}$ and have allowed faster commercialisation of ATMPs for life-threatening diseases ${ }^{10-12}$. The clinical development duration can be reduced from 6-7 years (phase 1, phase 2, phase 3 clinical trial) to 2-3 years (small scale clinical trials with surrogate end-points) $)^{14}$. Therefore, the commercialisation timeline for process, CMC development, and capacity planning decisions should also be considered earlier ${ }^{15}$.

In this work, we present a detailed analysis of the above challenges, and propose to develop an optimisation model to address the unique challenges of autologous cell therapies. The approach can potentially allow the industry to make better-informed investment decisions and accelerate the commercialisation of these life-saving therapies. 


\section{References}

1. Denault, J.-F., Coquet, A. \& Dodelet, V. Construction and Start-Up Costs for Biomanufacturing Plants Canadian Case Studies in the Cost of Regulatory Compliance.

https://bioprocessintl.com/wp-content/uploads/2014/05/BPI_A_080602AR02_O_76491a.pdf (2008).

2. Galambos, L. \& Sturchio, J. L. Pharmaceutical Firms and the Transition to Biotechnology: A Study in Strategic Innovation. Bus. Hist. Rev. 72, 250-278 (1998).

3. Rader, R. Cell and Gene Therapies: Industry Faces Potential Capacity Shortages. https://www.genengnews.com/gen-articles/cell-and-gene-therapies-industry-facespotential-capacity-shortages/6203 (2017).

4. Gatica, G., Papageorgiou, L. G. \& Shah, N. Capacity Planning Under Uncertainty for the Pharmaceutical Industry. Chem. Eng. Res. Des. 81, 665-678 (2003).

5. Papageorgiou, L., Rotstein, G. \& Shah, N. Strategic supply chain optimization for the pharmaceutical industries. Ind. Eng. Chem. Res. 40, 275-286 (2001).

6. Liu, S. \& Papageorgiou, L. G. Multiobjective optimisation of production, distribution and capacity planning of global supply chains in the process industry. (2012) doi:10.1016/j.omega.2012.03.007.

7. Siganporia, C. C., Ghosh, S., Daszkowski, T., Papageorgiou, L. G. \& Farid, S. S. Capacity planning for batch and perfusion bioprocesses across multiple biopharmaceutical facilities. Biotechnol. Prog. 30, 594-606 (2014).

8. Palmer, E. Dendreon, done in by expensive, complex manufacturing, files for bankruptcy. FiercePharma https://www.fiercepharma.com/supply-chain/dendreon-done-by-expensivecomplex-manufacturing-files-for-bankruptcy (2014).

9. Harrison, R. P., Rafiq, Q. A. \& Medcalf, N. Centralised versus decentralised manufacturing and the delivery of healthcare products: A United Kingdom exemplar. (2018) doi:10.1016/j.jcyt.2018.05.003.

10. Corrigan-Curay, J., McKee, A. E. \& Stein, P. Breakthrough-Therapy Designation - An FDA Perspective. N. Engl. J. Med. 378, 1457-1458 (2018).

11. Detela, G. \& Lodge, A. EU Regulatory Pathways for ATMPs: Standard, Accelerated and Adaptive Pathways to Marketing Authorisation. Molecular Therapy - Methods and Clinical Development vol. 13 205-232 (2019).

12. Kondo, H., Hata, T., Ito, K., Koike, H. \& Kono, N. The Current Status of Sakigake Designation in Japan, PRIME in the European Union, and Breakthrough Therapy Designation in the United States. Ther. Innov. Regul. Sci. 51, 51-54 (2017).

13. Vaggelas, A. \& Seimetz, D. Expediting Drug Development: FDA's New Regenerative Medicine Advanced Therapy Designation. Ther. Innov. Regul. Sci. 53, 364-373 (2019).

14. Somerville, S. \& Kloda, J. H. FDA's Expedited Review Process: The Need for Speed. Appl. Clin. Trials 24, 17-18 (2015).

15. Woodcock, J. \& Woosley, R. The FDA Critical Path Initiative and Its Influence on New Drug Development*. Annu. Rev. Med 59, 1-12 (2008). 